\title{
Electron Tomographic Comparison of Frozen-Hydrated and Freeze-Substituted Sections of High-Pressure Frozen Rat-Liver Tissue
}

\author{
C.-E Hsieh, ${ }^{*}$ M. Marko, ${ }^{*}$ A. Leith, ${ }^{*}$ J. Frank***, and C.A. Mannella,* \\ *Resource for Visualization of Biological Complexity, Wadsworth Center, Albany, NY 12201 \\ **Howard Hughes Medical Institute, Wadsworth Center, Empire State Plaza, Albany, NY 12201
}

High-pressure freezing is an ideal method for preparing tissue specimens in a "near-native" state. Chemical pre-fixation is not necessary, the water is rapidly frozen in the vitreous state, and formation of ice crystals that cause ultrastructural damage is suppressed to depths of well over 100 micrometers $[1,2]$. After high-pressure freezing, the tissue can be sectioned at low temperature, and tomography can be carried out on frozen-hydrated sections, avoiding the need for any dehydration or staining [3]. Alternatively, freeze-substitution can be employed in which post-fixation and dehydration are performed at low temperature, with the tissue finally being embedded in resin and sectioned at room temperature. There are numerous protocols for high-pressure freezing / freezesubstitution, depending on the specimen used and the staining qualities desired [e.g. 4,5].

Use of frozen-hydrated sections has the advantages of simplicity and the promise of preservation of native structure, since there is no tissue processing after freezing. Sectioning frozen tissue is challenging, since the sections must be handled "dry", and must be kept below the de-vitrification temperature $\left(\sim-140^{\circ} \mathrm{C}\right)$ to avoid formation of ice crystals. Sectioning causes about $30 \%$ compression in the direction parallel to the cutting direction, accompanied by an increase in thickness, but the means to overcome this are under development [6]. Surface artifacts associated with frozenhydrated sections do not extend to the interior [3]. Tomography of frozen-hydrated sections follows the same protocols that have been worked out for frozen-hydrated suspension layers [7]. Use of automation for collection of the tilt series is essential, since the total electron dose must be kept under $\sim 5000 \mathrm{e}^{-} / \mathrm{nm}^{2}$ in order to prevent distortion or movement of the section [3]. With frozen hydrated specimens, the attainable tomographic resolution ultimately depends on the total electron dose and on contrast; at liquid nitrogen temperature about $4 \mathrm{~nm}$ resolution can be achieved [8]. Further improvements in imaging, and protection of the specimen from radiation damage by cooling to liquid helium temperature may extend resolution to $2 \mathrm{~nm}$ [9].

The freeze-substitution approach has the advantage that sections are observed at room temperature and have high contrast due to en bloc and/or post-staining. For electron tomography, this high contrast, and the irradiation protection afforded to the specimen by the resin embedment, means that electron dose is not the limiting factor in tomographic resolution. Instead, the granularity of the stain and/or embedding resin limits the resolution, probably to $2 \mathrm{~nm}$. However, when taking advantage of the higher dose tolerated by the fine structure, mass loss from the resin causes the sections to collapse or compress about $30 \%$ in thickness. Pre-irradiation must be used so that the mass loss is completed before tomography begins.

For a particular biological application, it is important to determine whether the improvements in preservation and detection of fine structure using frozen-hydrated sections justifies the use of the more difficult technique. A commonly expressed goal of electron tomography is to locate and identify, within a cell, macromolecular complexes whose structures have previously been determined at high resolution by cryo-EM or x-ray crystallography [10]. For this application, frozen-hydrated sections offer the best chance of success for cells in their native environment within tissue. However, while frozen-hydrated sections offer access to potentially "more native" structure, 
obstacles to high resolution must still be assessed and overcome. In some types of studies, the advantages of freeze-substitution (improved contrast and radiation tolerance) could prove decisive. By this comparative study of frozen-hydrated and frozen-substituted sections, we hope to determine the practical advantages and limitations of each approach.

References

[1] H. Moor, in: Cryotechniques in Biological Electron Microscopy, R.A. Steinbrecht and K. Zierold, eds., Springer, Berlin (1987) 175.

[2] D. Studer et al., Scanning Microsc. Suppl. 3 (1989) 253.

[3] C.-E. Hsieh et al., J. Struct Biol. 138 (2002) 63.

[4] K. McDonald and M.K. Morphew, Microsc. Res. Tech. 24 (1993) 465.

[5] D. Studer et al., J. Microsc. 203 (2001) 285.

[6] D. Studer and H. Gnägi, J. Microsc. 197 (2000) 94.

[7] A.J. Koster et al., J. Struct. Biol. 120 (1997) 276.

[8] B.F. McEwen et al., J. Struct. Biol. 138 (2002) 47.

[9] J.M. Plitzko et al., Trends Biotech. 20 (2002) S40.

[10] A.S. Frangakis et al., PNAS 99 (2002) 14153.

[11] Supported by NIH NCRR Biomedical Research Technology Program Grant RR01219.
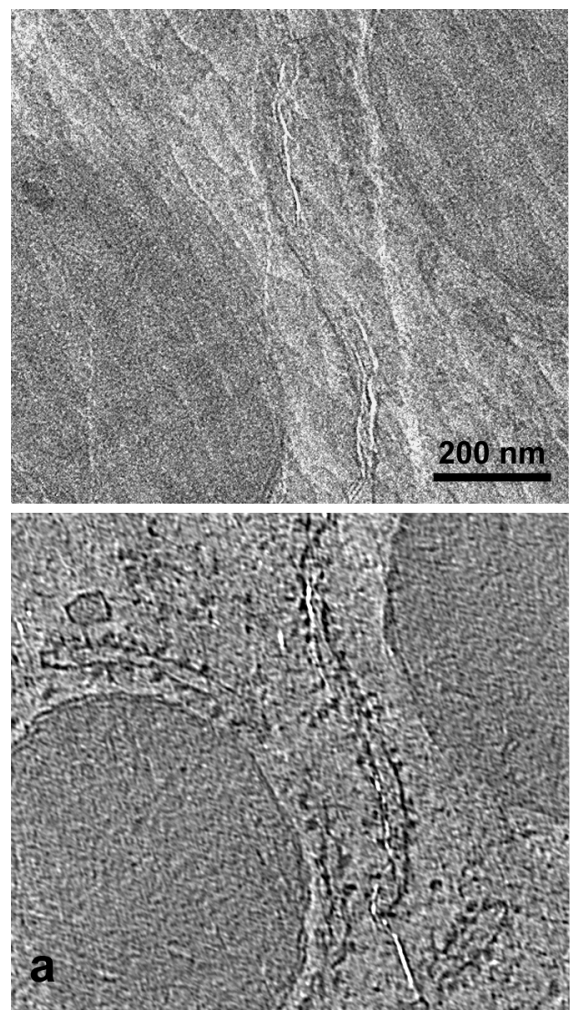
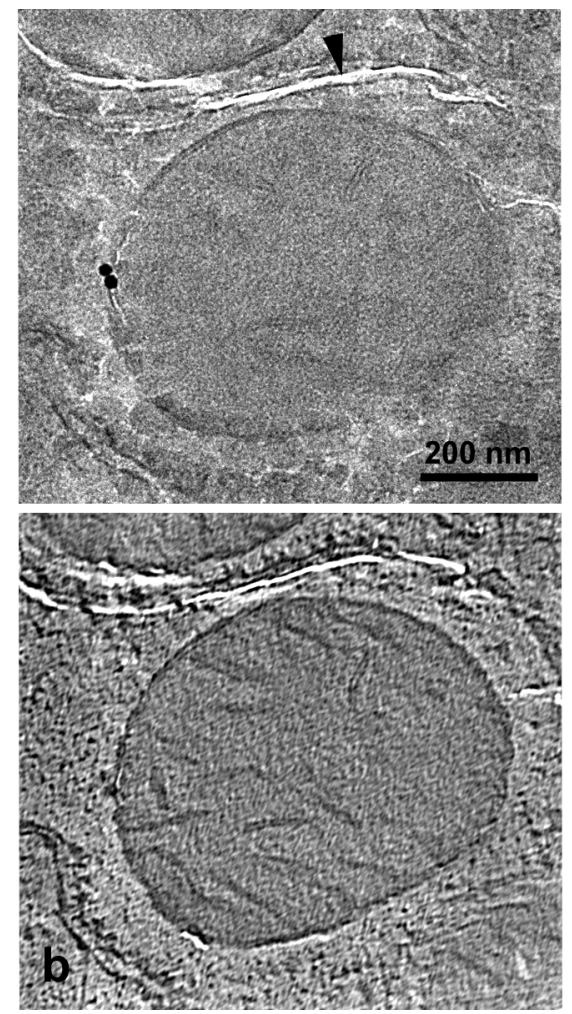
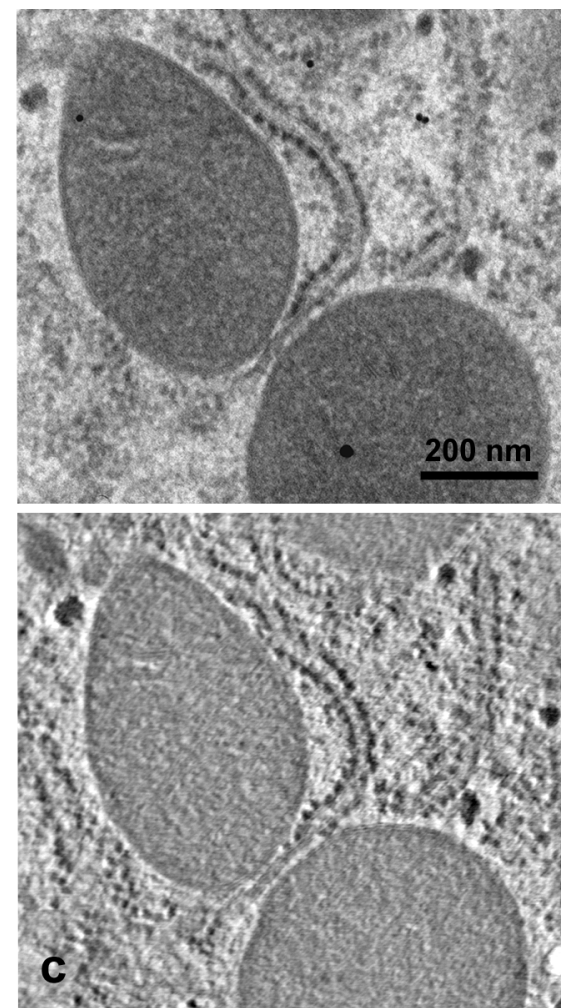

FIG. 1. Sections of rat liver tissue. Section thickness $150 \mathrm{~nm}$ (top). Tomographic slice thickness $1.7 \mathrm{~nm}$ (bottom). (a,b): Frozen-hydrated sections imaged at $400 \mathrm{kV}$ with zero-loss imaging using JEOL 4000FX and Gatan GIF 2002. Total dose $5000 \mathrm{e}^{-/ \mathrm{nm}^{2}}$. Crack in ice following ER at arrow. Note ribosomes on ER in (a) and mitochondrial cristae in (b). (c): Stained plastic section from tissue freeze-substituted with osmium. Conventional imaging at $120 \mathrm{kV}$. 